\title{
Odd Crossing Number and Crossing Number Are Not the Same
}

\author{
Michael J. Pelsmajer • Marcus Schaefer • \\ Daniel Štefankovič
}

Received: 28 September 2005 / Revised: 15 December 2005

(C) Springer Science+Business Media, LLC 2008

\begin{abstract}
The crossing number of a graph is the minimum number of edge intersections in a plane drawing of a graph, where each intersection is counted separately. If instead we count the number of pairs of edges that intersect an odd number of times, we obtain the odd crossing number. We show that there is a graph for which these two concepts differ, answering a well-known open question on crossing numbers. To derive the result we study drawings of maps (graphs with rotation systems).
\end{abstract}

\section{A Confusion of Crossing Numbers}

Intuitively, the crossing number of a graph is the smallest number of edge crossings in any plane drawing of the graph. As it turns out, this definition leaves room for interpretation, depending on how we answer the questions: what is a drawing, what is a crossing, and how do we count crossings? The papers by Pach and Tóth [7] and Székely [9] discuss the historical development of various interpretations and definitions - often implicit—of the crossing number concept.

A drawing $D$ of a graph $G$ is a mapping of the vertices and edges of $G$ to the Euclidean plane, associating a distinct point with each vertex, and a simple plane curve with each edge so that the ends of an edge map to the endpoints of the corresponding curve. For simplicity, we also require that

M.J. Pelsmajer ( $\varangle)$

Department of Applied Mathematics, Illinois Institute of Technology, Chicago, IL 60616, USA

e-mail: pelsmajer@iit.edu

M. Schaefer

Department of Computer Science, DePaul University, Chicago, IL 60604, USA

e-mail: mschaefer@cs.depaul.edu

D. Štefankovič

Computer Science Department, University of Rochester, Rochester, NY 14627-0226, USA

e-mail: stefanko@cs.rochester.edu 
- A curve does not contain any endpoints of other curves in its interior

- Two curves do not touch (that is, intersect without crossing), and

- No more than two curves intersect in a point (other than at a shared endpoint)

In such a drawing the intersection of the interiors of two curves is called a crossing. Note that by the restrictions we placed on a drawing, crossings do not involve endpoints, and at most two curves can intersect in a crossing. We often identify a drawing with the graph it represents. For a drawing $D$ of a graph $G$ in the plane we define

- $\operatorname{cr}(D)$ - the total number of crossings in $D$

- $\operatorname{pcr}(D)$ - the number of pairs of edges which cross at least once; and

- $\operatorname{ocr}(D)$ - the number of pairs of edges which cross an odd number of times

Remark 1 For any drawing $D$, we have $\operatorname{ocr}(D) \leq \operatorname{pcr}(D) \leq \operatorname{cr}(D)$.

We let $\operatorname{cr}(G)=\min \operatorname{cr}(D)$, where the minimum is taken over all drawings $D$ of $G$ in the plane. We define $\operatorname{ocr}(G)$ and $\operatorname{pcr}(G)$ analogously.

Remark 2 For any graph $G$, we have $\operatorname{ocr}(G) \leq \operatorname{pcr}(G) \leq \operatorname{cr}(G)$.

The question (first asked by Pach and Tóth [7]) is whether the inequalities are actually equalities. ${ }^{1}$ Pach [6] called this "perhaps the most exciting open problem in the area." The only evidence for equality is an old theorem by Chojnacki, which was later rediscovered by Tutte-and the absence of any counterexamples.

Theorem 1.1 (Chojnacki [4], Tutte [10]) If $\operatorname{ocr}(G)=0$, then $\operatorname{cr}(G)=0 .^{2}$

In this paper we will construct a simple example of a graph with $\operatorname{ocr}(G)<$ $\operatorname{pcr}(G)=\operatorname{cr}(G)$. We derive this example from studying what we call weighted maps on the annulus. Section 2 introduces the notion of weighted maps on arbitrary surfaces and gives a counterexample to $\operatorname{ocr}(M)=\operatorname{pcr}(M)$ for maps on the annulus. In Section 3 we continue the study of crossing numbers for weighted maps, proving in particular that $\operatorname{cr}(M) \leq c_{n} \cdot \operatorname{ocr}(M)$ for maps on a plane with $n$ holes. One of the difficulties in dealing with the crossing number is that it is NP-complete [2]. In Section 4 we show that the crossing number can be computed in polynomial time for maps on the annulus. Finally, in Section 5 we show how to translate the map counterexample from Section 2 into an infinite family of simple graphs for which $\operatorname{ocr}(G)<\operatorname{pcr}(G)$.

\section{Map Crossing Numbers}

A weighted map $M$ is a surface $S$ and a set $P=\left\{\left(a_{1}, b_{1}\right), \ldots,\left(a_{m}, b_{m}\right)\right\}$ of pairs of distinct points on $\partial S$ with positive weights $w_{1}, \ldots, w_{m}$. A realization $R$ of the map

\footnotetext{
${ }^{1}$ Doug West lists the problem on his page of open problems in graph theory [12]. Dan Archdeacon even conjectured that equality holds [1].

${ }^{2}$ In fact they proved something stronger, namely that in any drawing of a non-planar graph there are two non-adjacent edges that cross an odd number of times. Also see [8].
} 
Fig. 1 Optimal drawings: pcr and cr (above left), ocr (above right)

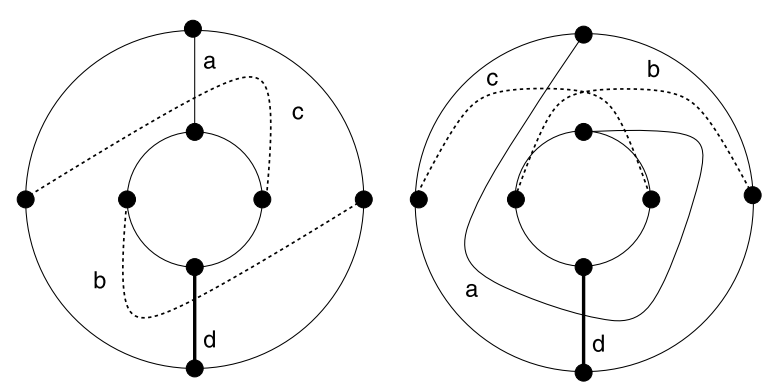

$M=(S, P)$ is a set of $m$ properly embedded $\operatorname{arcs} \gamma_{1}, \ldots, \gamma_{m}$ in $S$ where $\gamma_{i}$ connects $a_{i}$ and $b_{i}{ }^{3}$

Let

$$
\begin{aligned}
\operatorname{cr}(R) & =\sum_{1 \leq k<\ell \leq m} \iota\left(\gamma_{k}, \gamma_{\ell}\right) w_{k} w_{\ell}, \\
\operatorname{pcr}(R) & =\sum_{1 \leq k<\ell \leq m}\left[\iota\left(\gamma_{k}, \gamma_{\ell}\right)>0\right] w_{k} w_{\ell}, \\
\operatorname{ocr}(R) & =\sum_{1 \leq k<\ell \leq m}\left[\iota\left(\gamma_{k}, \gamma_{\ell}\right) \equiv 1(\bmod 2)\right] w_{k} w_{\ell},
\end{aligned}
$$

where $\iota\left(\gamma, \gamma^{\prime}\right)$ is the geometric intersection number of $\gamma$ and $\gamma^{\prime}$ and $[x]$ is 1 if the condition $x$ is true, and 0 otherwise. We define $\operatorname{cr}(M)=\min \operatorname{cr}(R)$, where the minimum is taken over all realizations $R$ of $M$. We define $\operatorname{pcr}(M)$ and $\operatorname{ocr}(M)$ analogously.

Remark 3 For every map $M, \operatorname{ocr}(M) \leq \operatorname{pcr}(M) \leq \operatorname{cr}(M)$.

Conjecture 1 For every map $M, \operatorname{cr}(M)=\operatorname{pcr}(M)$.

Lemma 2.1 If Conjecture 1 is true, then $\operatorname{cr}(G)=\operatorname{pcr}(G)$ for every graph $G$.

Proof Let $D$ be a drawing of $G$ with minimal pair crossing number. Drill small holes at the vertices. We obtain a drawing $R$ of a weighted map $M$. If Conjecture 1 is true, there exists a drawing of $M$ with the same crossing number. Collapse the holes to vertices to obtain a drawing $D^{\prime}$ of $G$ with $\operatorname{cr}\left(D^{\prime}\right) \leq \operatorname{pcr}(G)$.

However, we show below that we can separate the odd crossing number from the crossing number for weighted maps, even in the annulus (a disk with a hole).

When analyzing crossing numbers of drawings on the annulus, we describe curves with respect to an initial drawing of the curve and a number of Dehn twists. Consider, for example, the four curves in the left part of Figure 1. Comparing them to the

\footnotetext{
${ }^{3}$ If we take a realization $R$ of a map $M$, and contract each boundary component to a vertex, we obtain a drawing of a graph with a given rotation system [3]. For our purposes, maps are a more visual way to look at graphs with a rotation system. 
corresponding curves in the right part, we see that the curves labeled $c$ and $d$ have not changed, but the curves labeled $a$ and $b$ have each undergone a single clockwise twist.

Two curves are isotopic rel boundary if they can be obtained from each other by a continuous deformation which does not move the boundary $\partial M$. Isotopy rel boundary is an equivalence relation, its equivalence classes are called isotopy classes. An isotopy class on the annulus is determined by a properly embedded arc connecting the endpoints, together with the number of twists performed.

Lemma 2.2 Let $a \leq b \leq c \leq d$ be such that $a+c \geq d$. For the weighted map $M$ in Figure 1 we have $\operatorname{cr}(M)=\operatorname{pcr}(M)=a c+b d$ and $\operatorname{ocr}(M)=b c+a d$.

Proof The upper bounds follow from the drawings in Figure 1, the left drawing for crossing and pair crossing number, the right drawing for odd crossing number.

Claim $\operatorname{pcr}(M) \geq a c+b d$.

Proof of the Claim Let $R$ be a drawing of $M$ minimizing $\operatorname{pcr}(R)$. We can apply twists so that the thick edge $d$ is drawn as in the left part of Figure 1. Let $\alpha, \beta, \gamma$ be the number of clockwise twists applied to the ends of arcs $a, b, c$ on the inner boundary to obtain the drawing $R$, where $\alpha=\beta=\gamma=0$ corresponds to the drawing shown in the left part of Figure 1. Then,

$$
\begin{aligned}
\operatorname{pcr}(R)= & c d[\gamma \neq 0]+b d[\beta \neq-1]+a d[\alpha \neq 0]+b c[\beta \neq \gamma] \\
& +a b[\alpha \neq \beta]+a c[\alpha \neq \gamma+1] .
\end{aligned}
$$

If $\gamma \neq 0$, then $\operatorname{pcr}(R) \geq c d+a b$ because at least one of the last five conditions in (1) must be true; the last five terms contribute at least $a b$ (since $d \geq c \geq b \geq a$ ), and the first term contributes $c d$. Since $d(c-b) \geq a(c-b), c d+a b \geq a c+b d$, and the claim is proved in the case that $\gamma \neq 0$.

Now assume that $\gamma=0$. Equation (1) becomes

$$
\operatorname{pcr}(R)=b d[\beta \neq-1]+b c[\beta \neq 0]+a d[\alpha \neq 0]+a c[\alpha \neq 1]+a b[\alpha \neq \beta] .
$$

If $\beta \neq-1$, then $\operatorname{pcr}(R) \geq b d+a c$ because either $\alpha \neq 0$ or $\alpha \neq 1$. Since $b d+a c \geq$ $b c+a d$, the claim is proved in the case that $\beta \neq-1$.

This leaves us with the case that $\beta=-1$. Equation (2) becomes

$$
\operatorname{pcr}(R)=b c+a d[\alpha \neq 0]+a c[\alpha \neq 1]+a b[\alpha \neq-1] .
$$

The right-hand side of Equation (3) is minimized for $\alpha=0$. In this case $\operatorname{pcr}(R)=$ $b c+a c+a b \geq a c+b d$ because we assume that $a+c \geq d$.

Claim $\operatorname{ocr}(M) \geq b c+a d$.

Proof of the Claim Let $R$ be a drawing of $M$ minimizing ocr $(R)$. Let $\alpha, \beta, \gamma$ be as in the previous claim. We have 
$\operatorname{ocr}(R)=c d[\gamma]_{2}+b d[\beta+1]_{2}+a d[\alpha]_{2}+b c[\beta+\gamma]_{2}+a b[\alpha+\beta]_{2}+a c[\alpha+\gamma+1]_{2}$,

where $[x]_{2}$ is 0 if $x \equiv 0(\bmod 2)$, and 1 otherwise.

If $\beta \not \equiv \gamma(\bmod 2)$, then the claim clearly follows unless $\gamma=0, \beta=1$, and $\alpha=0$ (all modulo 2). In that case $\operatorname{ocr}(R) \geq b c+a b+a c \geq b c+a d$. Hence, the claim is proved if $\beta \not \equiv \gamma(\bmod 2)$.

Assume then that $\beta \equiv \gamma(\bmod 2)$. Equation $(4)$ becomes

$$
\operatorname{ocr}(R)=c d[\beta]_{2}+b d[\beta+1]_{2}+a d[\alpha]_{2}+a b[\alpha+\beta]_{2}+a c[\alpha+\beta+1]_{2} .
$$

If $\alpha \equiv 1(\bmod 2)$, then the claim clearly follows because either $c d$ or $b d$ contributes to the ocr. Thus we can assume $\alpha \equiv 0(\bmod 2)$. Equation $(5)$ becomes

$$
\operatorname{ocr}(R)=(c d+a b)[\beta]_{2}+(b d+a c)[\beta+1]_{2} .
$$

For both $\beta \equiv 0(\bmod 2)$ and $\beta \equiv 1(\bmod 2)$ we get $\operatorname{ocr}(R) \geq b c+a d$.

We get a separation of pcr and ocr for maps with small integral weights.

Corollary 2.3 There is a weighted map $M$ on the annulus with edges of weight $a=1$, $b=c=3$, and $d=4$ for which $\operatorname{cr}(M)=\operatorname{pcr}(M)=15$ and $\operatorname{ocr}(M)=13$.

Optimizing the gap over the reals yields $b=c=1, a=(\sqrt{3}-1) / 2$, and $d=1+a$, giving us the following separation of $\operatorname{pcr}(M)$ and $\operatorname{ocr}(M)$.

Corollary 2.4 There exists a weighted map $M$ on the annulus with $\operatorname{ocr}(M) \leq$ $\sqrt{3} / 2 \operatorname{pcr}(M)$.

Conjecture 2 For every weighted map $M$ on the annulus, $\operatorname{ocr}(M) \geq \frac{\sqrt{3}}{2} \operatorname{pcr}(M)$.

\section{Upper Bounds on Crossing Numbers}

In Section 5 we will transform the separation of ocr and pcr on maps into a separation on graphs. In particular, we will show that for every $\varepsilon>0$ there is a graph $G$ so that

$$
\operatorname{ocr}(G)<(\sqrt{3} / 2+\varepsilon) \operatorname{cr}(G)
$$

The gap cannot be arbitrarily large, as Pach and Tóth showed.

Theorem 3.1 (Pach and Tóth [7]) Let $G$ be a graph. Then $\operatorname{cr}(G) \leq 2(\operatorname{ocr}(G))^{2} .^{4}$

This result suggests the question whether the linear separation can be improved. We do not believe this to be possible:

\footnotetext{
${ }^{4}$ Better upper bounds on $\operatorname{cr}(G)$ in terms of $\operatorname{pcr}(G)$ are known [5, 11].

望 Springer
} 

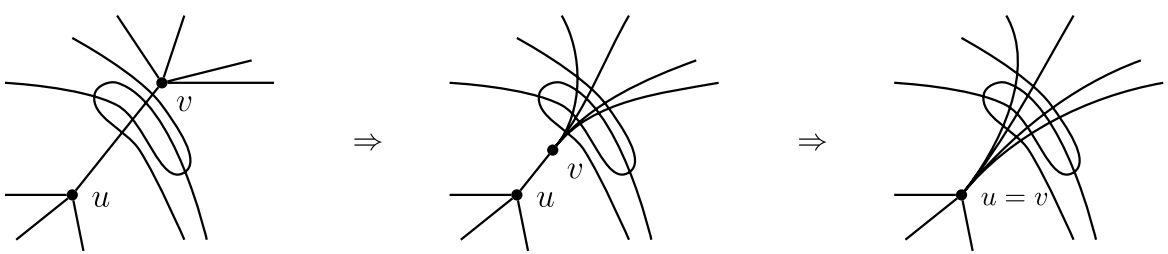

Fig. 2 Pulling an endpoint (left) and contracting the edge (right)

Conjecture 3 There is a $c>0$ so that $\operatorname{cr}(G)<c \cdot \operatorname{ocr}(G)$.

In this section, we will show that our approach of comparing the different crossing numbers for maps with a fixed number of holes will not lead to a super-linear separation. Namely, for a (weighted) map $M$ on a plane with $n$ holes, we always have

$$
\operatorname{cr}(M) \leq \operatorname{ocr}(M)\left(\begin{array}{c}
n+4 \\
4
\end{array}\right) / 5,
$$

with strict inequality if $n>1$. It follows that for fixed $n$, there $i$ s only a constant factor separating $\operatorname{cr}(M)$ and $\operatorname{ocr}(M)$. And only fixed, small $n$ are computationally feasible in analyzing potential counterexamples.

Observe that as a special case of Equation (7), if $M$ is a (weighted) map on the annulus $(n=2)$ we get that $\operatorname{cr}(M)<3 \operatorname{ocr}(M)$, which comes reasonably close to the $\sqrt{3} / 2$ lower bound from the previous section.

Before proving Equation (7) in full generality, we first consider the case of unit weights.

For this section only, we will switch our point of view from maps as curves between holes on a plane to maps as graphs with a rotation system; that is, we contract each hole to a vertex, and record the order, in which the curves (edges) leave the vertex. Our basic operation will be the contraction of an edge by pulling one of its endpoints along the edge, until it coincides with the other endpoint (the rotations of the vertices merge). Figure 2 illustrates pulling $v$ towards $u$ along $u v .^{5}$

Consider a drawing of $G$ with the minimum number of odd pairs (edge pairs that cross an odd number of times), $\operatorname{ocr}(G)$. We want to contract edges without creating too many new odd pairs. For each edge $e$, let $o_{e}$ be the number of edges that cross $e$ an odd number of times. Then $\sum_{e \in E(G)} o_{e}=2 \operatorname{ocr}(G)$, and since each edge is incident to exactly two vertices,

$$
\sum_{v \in V(G)} \sum_{e \ni v} o_{e}=4 \operatorname{ocr}(G) .
$$

Applying the pigeonhole principle twice, there must be a vertex $v \in V(G)$ with $\sum_{e \ni v} o_{e} \leq 4 \operatorname{ocr}(G) / n$, and there is a non-loop edge $e$ incident to $v$ with $o_{e} \leq$ $4 \operatorname{ocr}(G) /\left(n \cdot d^{*}(v)\right.$ ) (where $d^{*}(v)$ counts the number of non-loop edges incident to $v)$. Contracting $v$ to its neighbor along $e$ creates at most $o_{e}\left(d^{*}(v)-1\right)<$

\footnotetext{
${ }^{5}$ The illustration is taken from [8], where we investigate some other uses of this operation for graph drawings.
} 
$4 \operatorname{ocr}(G) / n$ odd pairs (only edges intersecting $e$ oddly will lead to odd intersections, and the parity of intersection along loops with endpoint $v$ does not change; selfintersections can be removed). Repeating this operation $n-1$ times, we transform $G$ into a bouquet of loops at a single vertex with at most $\operatorname{ocr}(G) \prod_{i=0}^{n-2}(1+4 /(n-i))$ odd pairs (strictly less if $n>1$ ). Without changing the rotation of the vertex, we can redraw all loops so that each odd pair intersects exactly once, and other pairs do not cross at all. We can then undo the contractions of the edges in reverse order without creating any new crossings. This yields a drawing of $G$ with the original vertex rotations with at most $\operatorname{ocr}(G) \prod_{i=0}^{n-2}(1+4 /(n-i))$ crossings. Since the product term equals $\left(\begin{array}{c}n+4 \\ 4\end{array}\right) / 5$, we have shown that $\operatorname{cr}(G) \leq \operatorname{ocr}(G)\left(\begin{array}{c}n+4 \\ 4\end{array}\right) / 5$ (strict inequality for $n>1$ ), as desired.

This argument proves Equation (7) for maps with unit weights. The next step is to extend this lemma to maps with arbitrary weights.

Consider two curves $\gamma_{1}, \gamma_{2}$ whose endpoints are adjacent and in the same order. In a drawing minimizing one of the crossing numbers we can always assume that the two curves are routed in parallel, following the curve that minimizes the total number of intersections with all curves other than $\gamma_{1}$ and $\gamma_{2}$. The same argument holds for a block of curves with adjacent endpoints in the same order. This allows us to claim Equation (7) for maps with integer weights: a curve with integral weight $w$ is replaced by $w$ parallel duplicates of unit weight.

If we scale all the weights in a map $M$ by a factor $\alpha$, all the crossing numbers will change by a factor of $\alpha^{2}$. Hence, the case of rational weights can be reduced to integer weights. Finally, we observe that if we consider any of the crossing numbers as a function of the weights of $M$, this function is continuous: This is obvious for a fixed drawing of $M$, so it remains true if we minimize over a finite set of drawings of $M$. The maximum difference in the number of twists in an optimal drawing is bounded by a function of the crossing number; and thus it suffices to consider a finite set of drawings of $M$. We have shown:

Theorem $3.2 \operatorname{cr}(M) \leq \operatorname{ocr}(M)\left(\begin{array}{c}n+4 \\ 4\end{array}\right) / 5$ for weighted maps $M$ on the plane with $n$ holes.

\section{Computing Crossing Numbers on the Annulus}

Let $M$ be a map on the annulus. We explained earlier that as far as crossing numbers are concerned, we can describe a curve in the realization of $M$ by a properly embedded arc $\gamma_{a b}$ connecting endpoints $a$ and $b$ on the inner and outer boundary of the annulus, and an integer $k \in \mathbb{Z}$, counting the number of twists applied to the curve $\gamma_{a b}$. Our goal is to compute the number of intersections between two arcs after applying a number of twists to each one of them. Since twists can be positive and negative and cancel each other out, we need to count crossings more carefully. Let us orient all arcs from the inner boundary to the outer boundary. Traveling along an arc $\alpha$, a crossing with $\beta$ counts as +1 if $\beta$ crosses from right to left, and as -1 if it crosses from left to right. Summing up these numbers over all crossings for two $\operatorname{arcs} \alpha$ and $\beta$ yields 
$\hat{\imath}(\alpha, \beta)$, the algebraic crossing number of $\alpha$ and $\beta$. Tutte [10] introduced the notion

$$
\operatorname{acr}(G)=\min _{D} \sum_{\{e, f\} \in\left(\begin{array}{c}
E \\
2
\end{array}\right)}\left|\hat{\imath}\left(\gamma_{e}, \gamma_{f}\right)\right|
$$

the algebraic crossing number of a graph, a notion that apparently has not drawn any attention since.

Let $D^{k}(\gamma)$ denote the result of adding $k$ twists to the curve $\gamma$. For two curves $\alpha$ and $\beta$ connecting the inner and outer boundary we have:

$$
\hat{\imath}\left(D^{k}(\alpha), D^{\ell}(\beta)\right)=k-\ell+\hat{\imath}(\alpha, \beta) .
$$

Note that $\iota(\alpha, \beta)=|\hat{\iota}(\alpha, \beta)|$ for any two curves $\alpha, \beta$ on the annulus.

Let $\pi$ be a permutation of $[n]$. A map $M_{\pi}$ corresponding to $\pi$ is constructed as follows. Choose $n+1$ points on each of the two boundaries and number them $0,1, \ldots, n$ in the clockwise order. Let $a_{i}$ be the vertex numbered $i$ on the outer boundary and $b_{i}$ be the vertex numbered $\pi_{i}$ on the inner boundary, $i=1, \ldots, n$. We ask $a_{i}$ to be connected to $b_{i}$ in $M_{\pi}$.

We will encode a drawing $R$ of $M_{\pi}$ by a sequence of $n$ integers $x_{1}, \ldots, x_{n}$ as follows. Fix a curve $\beta$ connecting the $a_{0}$ and $b_{0}$ and choose $\gamma_{i}$ so that $\iota\left(\beta, \gamma_{i}\right)=0$ (for all $i$ ). We will connect $a_{i}, b_{i}$ with the $\operatorname{arc} D^{x_{i}}\left(\gamma_{i}\right)$ in $R$. Note that for $i<j$, $\hat{\imath}\left(\gamma_{i}, \gamma_{j}\right)=\left[\pi_{i}>\pi_{j}\right]$ and hence

$$
\hat{\imath}\left(D^{x_{i}}\left(\gamma_{i}\right), D^{x_{j}}\left(\gamma_{j}\right)\right)=x_{i}-x_{j}+\left[\pi_{i}>\pi_{j}\right] .
$$

We have

$$
\begin{gathered}
\operatorname{acr}\left(M_{\pi}\right)=\operatorname{cr}\left(M_{\pi}\right)=\min \left\{\sum_{i<j}\left|x_{i}-x_{j}+\left[\pi_{i}>\pi_{j}\right]\right| w_{i} w_{j}: x_{i} \in \mathbb{Z}, i \in[n]\right\}, \\
\operatorname{pcr}\left(M_{\pi}\right)=\min \left\{\sum_{i<j}\left[x_{i}-x_{j}+\left[\pi_{i}>\pi_{j}\right] \neq 0\right] w_{i} w_{j}: x_{i} \in \mathbb{Z}, i \in[n]\right\},
\end{gathered}
$$

and

$$
\operatorname{ocr}\left(M_{\pi}\right)=\min \left\{\sum_{i<j}\left[x_{i}-x_{j}+\left[\pi_{i}>\pi_{j}\right] \not \equiv 0(\bmod 2)\right] w_{i} w_{j}: x_{i} \in \mathbb{Z}, i \in[n]\right\}
$$

Consider the relaxation of the integer program for $\operatorname{cr}\left(M_{\pi}\right)$ :

$$
\operatorname{cr}^{\prime}\left(M_{\pi}\right)=\min \left\{\sum_{i<j}\left|x_{i}-x_{j}+\left[\pi_{i}>\pi_{j}\right]\right| w_{i} w_{j}: x_{i} \in \mathbb{R}, i \in[n]\right\}
$$

Since (12) is a relaxation of (9), we have $\operatorname{cr}^{\prime}\left(M_{\pi}\right) \leq \operatorname{cr}\left(M_{\pi}\right)$. The following lemma shows that $\operatorname{cr}^{\prime}\left(M_{\pi}\right)=\operatorname{cr}\left(M_{\pi}\right)$. 
Lemma 4.1 Let $n$ be a positive integer. Let $b_{i j} \in \mathbb{Z}$ and let $a_{i j} \in \mathbb{R}$ be non-negative, $1 \leq i<j \leq n$. Then

$$
\min \left\{\sum_{i<j} a_{i j}\left|x_{i}-x_{j}+b_{i j}\right|: x_{i} \in \mathbb{R}, i \in[n]\right\}
$$

has an optimal solution with $x_{i} \in \mathbb{Z}, i \in[n]$.

Proof Let $\bar{x}^{*}$ be an optimal solution which satisfies the maximum number of $x_{i}-$ $x_{j}+b_{i j}=0,1 \leq i<j \leq n$. Without loss of generality, we can assume $x_{1}^{*}=0$. Let $G$ be a graph on the vertex set $\{1, \ldots, n\}$ with an edge between vertices $i, j$ if $x_{i}^{*}-x_{j}^{*}+b_{i j}=0$. Note that if $i, j$ are connected by an edge and one of $x_{i}^{*}, x_{j}^{*}$ is an integer, then both $x_{i}^{*}$ and $x_{j}^{*}$ are integers. It is then enough to show that $G$ is connected.

Suppose that $G$ is not connected. There exists a non-empty $A \subsetneq V(G)$ so that there are no edges between $A$ and $V(G)-A$. Let $\chi_{A}$ be the characteristic vector of the set $A$, that is, $\left(\chi_{A}\right)_{i}=[i \in A]$. Let $f(\lambda)$ be the value of the objective function on $\bar{x}=$ $\bar{x}^{*}+\lambda \cdot \chi_{A}$. Let $I$ be the interval on which the signs of the $x_{i}-x_{j}+b_{i j}, 1 \leq i<j \leq n$ are the same as for $\bar{x}^{*}$. Then $I$ is not the entire line (otherwise $G$ would be connected). Since $f$ is linear on $I, f$ is optimal at $\lambda=0$, and $I$ contains a neighborhood of 0 , it must be that $f$ is constant on $I$. Choosing $x=x^{*}+\lambda \chi_{A}$ for $\lambda$ an endpoint of $I$ gives an optimal solution satisfying more $x_{i}-x_{j}+b_{i j}=0,1 \leq i<j \leq n$, a contradiction.

Theorem 4.2 The crossing number of maps on the annulus can be computed in polynomial time.

Proof Note that $\mathrm{cr}^{\prime}\left(M_{\pi}\right)$ is computed by the following linear program $L_{\pi}$ :

$$
\begin{aligned}
\min & \sum_{i<j} y_{i j} w_{i} w_{j}, \\
& y_{i j} \geq x_{i}-x_{j}+\left[\pi_{i}>\pi_{j}\right], \quad 1 \leq i<j \leq n, \\
& y_{i j} \geq-x_{i}+x_{j}-\left[\pi_{i}>\pi_{j}\right], \quad 1 \leq i<j \leq n .
\end{aligned}
$$

Question 1 Let $M$ be a map on the annulus. Can $\operatorname{ocr}(M)$ be computed in polynomial time?

We conjectured earlier that crossing number and odd crossing number agree on maps. A more moderate goal would be to establish the following conjecture.

Conjecture 4 For any map $M$ on the annulus $\operatorname{cr}(M)=\operatorname{pcr}(M)$.

\section{Separating Crossing Numbers of Graphs}

We modify the map from Lemma 2.2 to obtain a graph $G$ separating $\operatorname{ocr}(G)$ and $\operatorname{pcr}(G)$. The graph $G$ will have integral weights on edges. From $G$ we can get an 
Fig. 3 The inside flipped

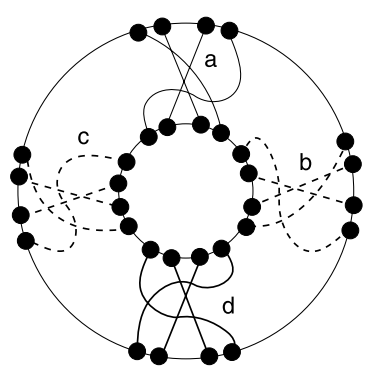

unweighted graph $G^{\prime}$ with $\operatorname{ocr}\left(G^{\prime}\right)=\operatorname{ocr}(G)$ and $\operatorname{pcr}\left(G^{\prime}\right)=\operatorname{pcr}(G)$ by replacing an edge of weight $w$ by $w$ parallel edges of weight 1 (this does not change any of the crossing numbers). If needed we can get rid of parallel edges by subdividing edges, which does not change any of the crossing numbers.

We start with the map $M$ from Lemma 2.2 with the following integral weights:

$$
a=\left\lfloor\frac{\sqrt{3}-1}{2} m\right\rfloor, \quad b=c=m, \quad d=\left\lfloor\frac{\sqrt{3}+1}{2} m\right\rfloor,
$$

where $m \in \mathbb{N}$ will be chosen later.

We replace each pair $\left(a_{i}, b_{i}\right)$ of $M$ by $w_{i}$ pairs $\left(a_{i, 1}, b_{i, 1}\right), \ldots,\left(a_{i, w_{i}}, b_{i, w_{i}}\right)$ where the $a_{i, j}\left(b_{i, j}\right)$ occur on $\partial S$ in clockwise order in a small interval around of $a_{i}\left(b_{i}\right)$. As before, we can argue that all the curves corresponding to $\left(a_{i}, b_{i}\right)$ can be routed in parallel in an optimal drawing and, therefore, the resulting map $N$ with unit weights will have the same crossing numbers as $M$.

We then replace the boundaries of the annulus by cycles (using one vertex for each $a_{i, j}$ and $b_{i, j}$, obtaining a graph $G$. We assign weight $W=1+\operatorname{pcr}(N)$ to the edges in the cycles. This ensures that in a drawing of $G$ minimizing any of the crossing numbers the boundary cycles are embedded without any intersections. Consequently, a drawing of $G$ on the sphere that minimizes any one of the crossing numbers looks very much like the drawing of a map on the annulus. With one subtle difference: one of the boundaries may flip.

Given the map $N$ on the annulus, the flipped map $N^{\prime}$ is obtained by flipping the order of the points on one of the boundaries. In other words, there are essentially two different ways of embedding the two boundary cycles of $G$ on the sphere without intersections depending on the relative orientation of the boundaries. In one of the cases the drawing $D$ of $G$ gives a drawing of $N$, in the other case it gives a drawing of the flipped map $N^{\prime}$. Fortunately, in the flipped case the group of edges corresponding to the weighted edge from $a_{i}$ to $b_{i}$ must intersect often with each other (as illustrated in Figure 3).

Now we know that

$$
\begin{aligned}
\operatorname{ocr}(G) & \leq \operatorname{ocr}(N) \quad(\text { since every drawing of } N \text { is a drawing of } G) \\
& \leq w_{1} w_{3}+w_{2} w_{4} \quad \text { (by Lemma 2.2) } \\
& \leq \frac{3}{2} m^{2} \quad \text { (by the choice of weights). }
\end{aligned}
$$


We will presently prove the following estimate on the flipped map.

Lemma $5.1 \operatorname{ocr}\left(N^{\prime}\right) \geq 2 m^{2}-4 m$.

With that estimate and our discussion of flipped maps, we have

$$
\begin{aligned}
\operatorname{pcr}(G) & =\min \left\{\operatorname{pcr}(N), \operatorname{pcr}\left(N^{\prime}\right)\right\} \\
& \geq \min \left\{\operatorname{pcr}(N), \operatorname{ocr}\left(N^{\prime}\right)\right\} \quad(\text { since ocr } \leq \mathrm{cr}) \\
& \geq \min \left\{\sqrt{3} m^{2}-2 m, 2 m^{2}-4 m\right\} \quad(\text { choice of } w, \text { and Lemma 5.1). }
\end{aligned}
$$

By making $m$ sufficiently large, we can make the ratio of $\operatorname{ocr}(G)$ and $\operatorname{pcr}(G)$ arbitrarily close to $\sqrt{3} / 2$.

Theorem 5.2 For any $\varepsilon>0$ there is a graph $G$ such that

$$
\operatorname{ocr}(G)<(\sqrt{3} / 2+\varepsilon) \operatorname{pcr}(G)
$$

The proof of Lemma 5.1 will require the following estimate.

Lemma 5.3 Let $0 \leq a_{1} \leq a_{2} \leq \cdots \leq a_{n}$ be such that $a_{n} \leq a_{1}+\cdots+a_{n-1}$. Then

$$
\max _{\left|y_{i}\right| \leq a_{i}}\left(\left(\sum_{i=1}^{n} y_{i}\right)^{2}-2 \sum_{i=1}^{n} y_{i}^{2}\right)=\left(\sum_{i=1}^{n} a_{i}\right)^{2}-2 \sum_{i=1}^{n} a_{i}^{2} .
$$

Proof of Lemma 5.1 Let $w_{1}=a, w_{2}=b, w_{3}=d, w_{4}=c$ (with $a, b, c, d$ as in the definition of $N$ ). In any drawing of $N^{\prime}$ each group of the edges split into two classes, those with an even number of twists and those with an odd number of twists (two twists make the same contribution to $\operatorname{ocr}\left(M^{\prime}\right)$ as no twists). Consequently, we can estimate $\operatorname{ocr}\left(N^{\prime}\right)$ as follows.

$$
\begin{aligned}
\operatorname{ocr}\left(N^{\prime}\right) & =\min _{k_{i} \in\left\{0,1, \ldots, w_{i}\right\}}\left(\sum_{i=1}^{4}\left(\begin{array}{c}
k_{i} \\
2
\end{array}\right)+\sum_{i=1}^{4}\left(\begin{array}{c}
w_{i}-k_{i} \\
2
\end{array}\right)+\sum_{i \neq j} k_{i}\left(w_{j}-k_{j}\right)\right) \\
& \geq-\frac{1}{2} \sum_{i=1}^{4} w_{i}+\min _{0 \leq x_{i} \leq w_{i}}\left(\sum_{i=1}^{4} \frac{x_{i}^{2}}{2}+\sum_{i=1}^{4} \frac{\left(w_{i}-x_{i}\right)^{2}}{2}+\sum_{i \neq j} x_{i}\left(w_{j}-x_{j}\right)\right) \\
& =-\frac{1}{2} \sum_{i=1}^{4} w_{i}+\frac{1}{4}\left(\sum_{i=1}^{4} w_{i}\right)^{2}+\min _{\left|y_{i}\right| \leq w_{i} / 2}\left(2 \sum_{i=1}^{4} y_{i}^{2}-\left(\sum_{i=1}^{4} y_{i}\right)^{2}\right) \\
& \geq \frac{1}{2} \sum_{i=1}^{4} w_{i}^{2}-\frac{1}{2} \sum_{i=1}^{4} w_{i}(\mathrm{using} \operatorname{Lemma~} 5.3) \\
& \geq \frac{1}{2}\left(\left(\frac{\sqrt{3}+1}{2} m-1\right)^{2}+2 m^{2}+\left(\frac{\sqrt{3}-1}{2} m-1\right)^{2}-4 m\right) \\
& \geq 2 m^{2}-4 m .
\end{aligned}
$$


The equality between the second and third line can be verified by substituting $y_{i}=$ $x_{i}-w_{i} / 2$.

Proof of Lemma 5.3 Let $y_{1}, \ldots, y_{n}$ achieve the maximum value. Replacing the $y_{i}$ by $\left|y_{i}\right|$ does not decrease the objective function. Without loss of generality, we can assume $0 \leq y_{1} \leq y_{2} \leq \cdots \leq y_{n}$. Note that if $y_{i}<y_{j}$, then $y_{i}=a_{i}$ (otherwise increasing $y_{i}$ by $\varepsilon$ and decreasing $y_{j}$ by $\varepsilon$ increases the objective function for small $\varepsilon$ ).

Let $k$ be the largest $i$ such that $y_{i}=a_{i}$. Let $k=0$ if no such $i$ exists. We have $y_{i}=a_{i}$ for $i \leq k$ and $y_{k+1}=\cdots=y_{n}$. If $k=n$ we are done.

We conclude the proof by showing that $k<n$ is not possible. Let $t$ be the common value of $y_{k+1}=\cdots=y_{n}$. Note that we have $t=y_{k+1} \leq a_{k+1}$.

Let

$$
f(t)=\left(\left(\sum_{i=1}^{k} a_{i}\right)+(n-k) t\right)^{2}-2\left(\left(\sum_{i=1}^{k} a_{i}^{2}\right)+(n-k) t^{2}\right)
$$

We have

$$
f^{\prime}(t)=2(n-k)\left(\left(\sum_{i=1}^{k} a_{i}\right)+(n-k-2) t\right) .
$$

Note that $f^{\prime}(t)>0$ for $t<a_{k+1}$. (This is easy to see when $k<n-1$; for $k=n-1$ we make use of the assumption that $a_{n} \leq \sum_{i=1}^{n-1} a_{i}$.) Therefore, $f(t)$ will be maximized by $t=a_{k+1}$ over values $t \leq a_{k+1}$. Hence, $y_{k+1}=a_{k+1}$, contradicting our choice of $k$.

\section{Conclusion}

The relationship between the different crossing numbers remains mysterious, and we have already mentioned several open questions and conjectures. Here we want to revive a question first asked by Tutte (in slightly different form). Recall the definition of the algebraic crossing number from Section 4:

$$
\operatorname{acr}(G)=\min _{D} \sum_{\{e, f\} \in\left(\begin{array}{c}
E \\
2
\end{array}\right)}\left|\hat{\imath}\left(\gamma_{e}, \gamma_{f}\right)\right|
$$

where $\gamma_{e}$ is a curve representing edge $e$ in a drawing $D$ of $G$. It is clear that

$$
\operatorname{acr}(G) \leq \operatorname{cr}(G)
$$

Does equality hold?

Acknowledgement Thanks to the anonymous referee for helpful comments. 


\section{References}

1. Archdeacon, D.: Problems in topological graph theory. http://www.emba.uvm.edu/ archdeac/ problems/altcross.html (accessed April 7th, 2005)

2. Garey, M.R., Johnson, D.S.: Crossing number is NP-complete. SIAM J. Algebr. Discrete Methods 4(3), 312-316 (1983)

3. Gross, J.L., Tucker, T.W.: Topological Graph Theory. Dover, Mineola (2001). Reprint of the 1987 original

4. Chojnacki, C. (Haim Hanani): Über wesentlich unplättbare Kurven im drei-dimensionalen Raume. Fundam. Math. 23, 135-142 (1934)

5. Kolman, P., Matoušek, J.: Crossing number, pair-crossing number, and expansion. J. Comb. Theory Ser. B 92(1), 99-113 (2004)

6. Pach, J.: Crossing numbers. In: Discrete and Computational Geometry (Tokyo, 1998). Lecture Notes in Comput. Sci., vol. 1763, pp. 267-273. Springer, Berlin (2000)

7. Pach, J., Tóth, G.: Which crossing number is it anyway? J. Comb. Theory Ser. B 80(2), 225-246 (2000)

8. Pelsmajer, M.J., Schaefer, M., Štefankovič, D.: Removing even crossings. In: EuroComb, April 2005

9. Székely, L.A.: A successful concept for measuring non-planarity of graphs: the crossing number. Discrete Math. 276(1-3), 331-352 (2004). 6th International Conference on Graph Theory

10. Tutte, W.T.: Toward a theory of crossing numbers. J. Comb. Theory 8, 45-53 (1970)

11. Valtr, P.: On the pair-crossing number. In: Combinatorial and Computational Geometry. MSRI Publications, vol. 52, pp. 545-551 (2005)

12. West, D.: Open problems—graph theory and combinatorics. http://www.math.uiuc.edu/ west/openp/ (accessed April 7th, 2005) 